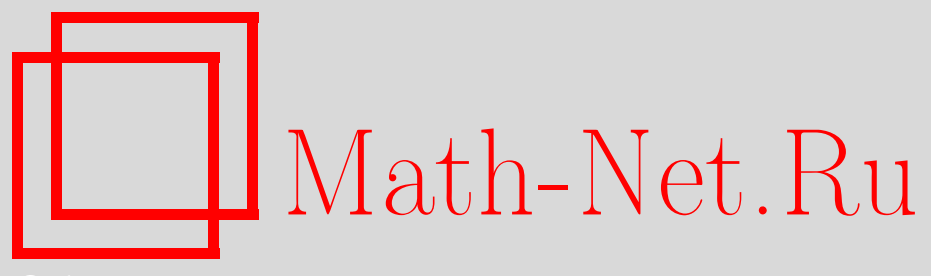

К. Бардос, Л. Секелихиди мл., Э. Видеманн, Об отсутствии единственности для уравнений Эйлера: эффект границы, УМН, 2014, том 69, выпуск 2, 3-22

DOI: https://doi.org/10.4213/rm9578

Использование Общероссийского математического портала Math-Net.Ru подразумевает, что вы прочитали и согласны с пользовательским соглашением http://www . mathnet.ru/rus/agreement

Параметры загрузки:

IP : 52.23 .180 .231

26 апреля 2023 г., 09:16:55

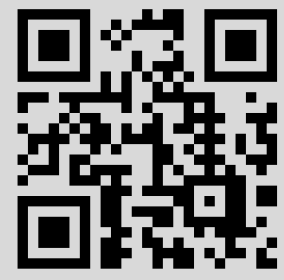




\title{
Об отсутствии единственности для уравнений Эйлера: эффект границы
}

\author{
К. Бардос, Л. Секелихиди мл., Э. Видеманн
}

Рассматриваются начальные данные вращательного типа для двумерных уравнений Эйлера динамики несжимаемой жидкости в круговом кольце. Используя методику выпуклого интегрирования, мы показываем, что существует бесконечно много допустимых слабых решений (т.е. решений с невозрастающей энергией) с этими начальными данными. Как следствие, в ограниченных областях существуют допустимые слабые решения, не являющиеся диссипативными по П.-Л. Лионсу, что составляет контраст со случаем, когда физические границы отсутствуют. Кроме того, показано, что допустимые решения являются диссипативными, если они обладают свойством Гёльдера вблизи границы области.

Библиография: 34 названия.

Ключевые слова: уравнения Эйлера, неединственность, дикие решения, диссипативные решения, эффект границы, выпуклое интегрирование, предел нулевой вязкости, потоки вращения.

DOI: $10.4213 / \mathrm{rm} 9578$

\section{СОДЕРЖАНИЕ}

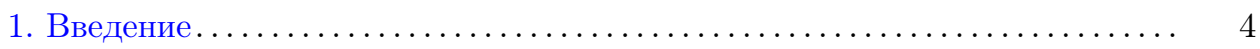

2. Формулировки основных результатов ...................... 5

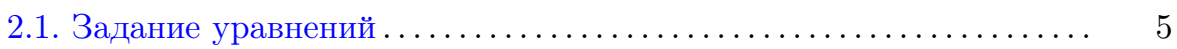

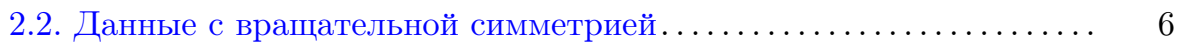

3. Субрешения и выпуклое интегрирование $\ldots \ldots \ldots \ldots \ldots \ldots \ldots \ldots \ldots . \ldots \ldots$

4. Неединственность для начальных данных вращательного типа . . .... 9

5. Единственность предела нулевой вязкости . . . . . . . . . . . . . . . 13

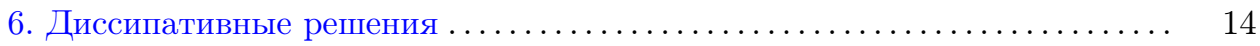

7. Критерий диссипативности допустимого решения ................ 16

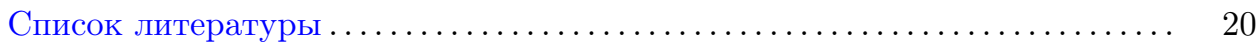

Работа второго автора поддержана Европейским советом по исследованиям (грант № 277993). Часть настоящей работы была выполнена в то время, когда третий автор был гостем проекта "Instabilities in Hydrodynamics" Фонда математических наук Парижа. Он благодарен Фонду за поддержку.

(С) К. БАрдос, Л. СЕКЕЛИХиди мЛ., Э. ВидЕманН, 2014 


\section{1. Введение}

Исследования слабых решений уравнений Эйлера динамики несжимаемой жидкости мотивированы (по меньшей мере) двумя свойствами потоков жидкости: наличием неустойчивости, а именно неустойчивости Кельвина-Гельмгольца, и развитой 3-мерной турбулентностью. Что касается второго аспекта, здесь возникает важная задача в связи со знаменитым “законом 5/3" Колмогорова-Обухова и гипотезой Онзагера относительно сохранения энергии. Тут мы сошлемся на работы [3], [15] и [6], [9], [19], где можно найти дополнительную информацию и узнать о достижениях последнего времени в решении этой задачи.

Что касается первого аспекта, то он активно анализировался с целью выработки определения слабого решения, учитывающего основные свойства подобных неустойчивостей и в то же время обладающего приемлемыми аналитическими свойствами. Поскольку здесь отсутствует аналог теоремы существования у уравнений Навье-Стокса слабых решений в смысле Лере-Хопфа, рассматривался ряд более слабых понятий.

Диссипативные решения уравнений Эйлера динамики несжимаемой жидкости были введены П.-Л. Лионсом [22] как класс решений с двумя желательными свойствами: (i) их существование для произвольных начальных данных и (ii) "единственность сильных решений в классе слабых", означающая, что диссипативное слабое решение совпадает с сильным, если последнее существует. Как было показано, диссипативные решения уравнений Эйлера несжимаемой жидкости возникают, в частности, как пределы нулевой вязкости [22] и гидродинамические пределы [25]. Главный недостаток диссипативных решений заключался в том, что, вообще говоря, их поле скоростей не является решением уравнений Эйлера в смысле распределений.

С другой стороны, слабые решения (т. е. решения в смысле распределений, обладающие некоторыми дополнительными свойствами) строятся различными методами; см. [10], [11], [26], [28], [29], [31], [32], [34]. Многим из них в высокой степени присуще отсутствие единственности, так что даже свойство единственности сильных решений в классе слабых не обязательно имеет место (здесь можно сослаться на обзор [12]). В частности, в [34] для любых начальных данных было установлено существование глобальных по времени слабых решений.

В условиях высокой степени неединственности возникает естественный вопрос, есть ли какие-нибудь критерии отбора слабых решений. По этому поводу в [11], [13] было замечено, что когда область не имеет границы, слабое решение будет диссипативным по Лионсу, если для него выполнено слабое энергетическое неравенство

$$
\int|v(x, t)|^{2} d x \leqslant \int|v(x, 0)|^{2} d x \text { для почти всех } t>0 .
$$

В [11] это условие называется условием допустимости, по аналогии с энтропийным условием, возникающим в гиперболических законах сохранения [8]. Допустимость оказалась полезным критерием отбора слабых решений, так как даже слабая форма (1) этого условия влечет единственность сильных решений 
в классе слабых, требуемую от диссипативных решений (более сильные варианты энергетического неравенства обсуждаются в [11]). Это верно не только для решений в смысле распределений, но даже для мерозначных решений (см. [5]).

Несмотря на единственность сильных решений в классе слабых, существует большое подмножество начальных данных (на самом деле всюду плотное в $L^{2}$ ), заданных на всем пространстве или в комбинации с периодическими граничными условиями [32] (см. также [31]), для которых начально-краевая задача имеет бесконечно много допустимых слабых решений. Такие начальные данные, называемые "дикими", не могут быть регулярными.

Неединственность допустимых слабых решений тесно связана с наличием неустойчивости. Например, в работе [30] неединственность допустимых слабых решений была продемонстрирована для начальных данных

$$
v_{0}(x)= \begin{cases}e_{1}, & \text { если } x_{d} \in\left(0, \frac{1}{2}\right), \\ -e_{1}, & \text { если } x_{d} \in\left(-\frac{1}{2}, 0\right),\end{cases}
$$

включающих плоскую вихревую поверхность и периодически продолженных на тор $\mathbb{T}^{d}$. Заметим, что в этом случае очевидно имеется стационарное решение, но результат [30] заключается в том, что есть еще бесконечно много нестационарных решений. Их общей чертой является существование для $t>0$ расширяющегося региона “турбулентности”, охватывающего начальную вихревую поверхность, что сильно напоминает распространение особенностей в классической задаче Кельвина-Гельмгольца. Другие подобные примеры были позднее найдены в [4], а также недавно в [7] для системы Эйлера динамики сжимаемой жидкости.

В настоящей статье, вдохновляемые идеей, что именно неустойчивость Кельвина-Гельмгольца лежит в основе неединственности допустимых слабых решений, мы исследуем класс областей с границей. Мы покажем, что наличие (гладкой) границы может иметь результатом тот же эффект расширяющегося региона турбулентности, что и в [30]. Как следствие, мы отмечаем, что в областях с границей допустимость не означает единственности сильных решений в классе слабых.

\section{2. Формулировки основных результатов}

2.1. Задание уравнений. Мы изучаем слабые решения начально-краевой задачи для уравнений Эйлера несжимаемой жидкости

$$
\begin{aligned}
\partial_{t} v+v \cdot \nabla v+\nabla p & =0, \\
\operatorname{div} v & =0, \\
\left.v\right|_{t=0} & =v_{0},
\end{aligned}
$$

дополненных кинематическим краевым условием

$$
\left.v\right|_{\partial \Omega} \cdot \nu=0
$$


Здесь $\Omega \subset \mathbb{R}^{d}, d \geqslant 2,-$ область с достаточно гладкой границей, $T>0$ конечный момент времени, $v: \Omega \times[0, T) \rightarrow \mathbb{R}^{d}-$ поле скоростей, $p: \Omega \times(0, T) \rightarrow$ $\mathbb{R}$ - (скалярное) давление, $v_{0}$ - начальная скорость, а $\nu$ - единичная внутренняя нормаль к границе $\Omega$.

Для точного определения слабых решений рассмотрим пространство соленоидальных векторных полей в $\Omega$ (ср. [16; гл. III]):

$$
\begin{aligned}
& H(\Omega)=\left\{v \in L^{2}\left(\Omega ; \mathbb{R}^{d}\right):\right. \int_{\Omega} v \cdot \nabla p d x=0 \\
&\text { для всех } \left.p \in W_{\text {lос }}^{1,2}(\Omega) \text { с } \nabla p \in L^{2}(\Omega)\right\} .
\end{aligned}
$$

Пусть $v_{0} \in H(\Omega)$. По определению, допустимое слабое решение системы (3) с начальными данными $v_{0}$ - это такое векторное поле $v \in L^{\infty}(0, T ; H(\Omega))$, что для всякой основной функции $\phi \in C_{c}^{\infty}\left(\Omega \times[0, T) ; \mathbb{R}^{d}\right)$ такой, что $\operatorname{div} \phi=0$, выполнено равенство

$$
\int_{0}^{T} \int_{\Omega}\left(\partial_{t} \phi \cdot v+\nabla \phi: v \otimes v\right) d x d t+\int_{\Omega} v_{0}(x) \cdot \phi(x, 0) d x=0
$$

а также энергетическое неравенство (1).

Заметим, кстати, что на самом деле допустимые слабые решения можно считать элементами пространства $C\left([0, T) ; H_{\mathrm{w}}(\Omega)\right)$, где $H_{\mathrm{w}}(\Omega)$ - это пространство $H(\Omega)$ со слабой $L^{2}$-топологией. И действительно, диссипативные решения по Лионсу являются элементами этого пространства. Тем не менее для простоты мы будем считать только, что поля скоростей являются элементами большего пространства $L^{\infty}(0, T ; H(\Omega))$.

2.2. Данные с вращательной симметрией. В статье рассматриваются двумерные начальные данные с вращательной симметрией. Отметим, что ограничение на размерность введено исключительно для простоты изложения - все конструкции и подходы легко распространить на более высокую размерность. Область в виде кругового кольца рассматривается также для простоты изложения - ее нетривиальная топология не играет никакой роли для результатов.

Под данными “вращательного типа" мы подразумеваем начальные данные вида

$$
v_{0}(x)=\alpha_{0}(r)(\sin \theta,-\cos \theta)
$$

в кольце

$$
\Omega=\left\{x \in \mathbb{R}^{2}: \rho<|x|<R\right\},
$$

где $0<\rho<R<\infty$. Как известно, для любого выбора $\alpha_{0}$ векторные поля (4) задают стационарные решения уравнений Эйлера; их часто приводят в качестве наглядных примеров при исследовании потоков несжимаемой жидкости [1], [24], [27]. 
Зафиксируем радиус $r_{0}$, где $\rho<r_{0}<R$, и рассмотрим начальные данные в кольце, описываемые соотношением (4), где

$$
\alpha_{0}(r)= \begin{cases}-\frac{1}{r^{2}}, & \text { если } \rho<r<r_{0}, \\ \frac{1}{r^{2}}, & \text { если } r_{0}<r<R,\end{cases}
$$

что соответствует потоку вращения со скачком на окружности $\left\{r=r_{0}\right\}$.

Теорема 1. Пусть $\Omega$ - круговое колъи, (5), $T>0$ - конечный момент времени, а $v_{0}$ имеет вращательный тип как в (4) и (6). Тогда помимо стаицонарного решения $v(\cdot, t)=v_{0}$ существует бесконечное количество нестационарных допустимых слабых решений уравнений Эйлера в $\Omega \times(0, T)$ с начальным значением $v_{0}$. У бесконечного множества решений из их числа энергия строго убывает и у бесконечного множества решений - сохраняется.

Доказательство, которое мы приводим ниже в разделе 4, использует методы из работы [11] и аналогично конструкции, приведенной в [30].

Что касается поиска подходящих принципов отбора, в литературе широко обсуждается отбор только вязкостных решений, т. е. решений, полученных как слабый предел решений Лере-Хопфа при стремлении вязкости к нулю. В случае начальных данных вида (2) легко проверить (к примеру, см. [4]), что вязкостное решение должно совпадать со стационарным. То же самое происходит в случае данных вращательного типа (6), что было отмечено, например, в [23] (где на самом деле был получен более глубокий результат для начальных данных в виде локализованного вихря). Для удобства читателей в разделе 5 мы приводим элементарное доказательство соответствующего результата.

ПредлОЖениЕ 2. Пусть $\Omega \subset \mathbb{R}^{2}$ - круговое кольцо, и пусть начальные данные задаются выражением (4). Тогда любал последовательность решений Лере-Хопфа уравнений Навъе-Стокса с вязкостью, стремящейся к нулю, соответствующая тем же начальным данным, сходится в сильном смысле $\kappa$ стационарному решению $v(\cdot, t)=v_{0}$ уравнений Эйлера.

В заключение обсудим связь между допустимыми слабыми решениями и диссипативными решениями Лионса в ограниченных областях. Для удобства читателей в разделе 6 мы напоминаем точное определение диссипативных решений. В качестве следствия из теоремы 1 мы покажем в разделе 6, что, в отличие от ситуации, когда границы нет, допустимые слабые решения не обязательно диссипативны.

СлеДСтвиЕ 3. В $\Omega$ существуют допустимые слабые решения, не являющиеся диссипативными решениями.

Следствие 3 говорит о том, что в присутствии границы для допустимых слабых решений не обязательно выполнено свойство единственности сильных решений в классе слабых. На формальном уровне это объясняется тем, что у сильного решения в ограниченной области мы никак не можем проконтролировать граничное поведение. По этой причине в разделе 7 мы исследуем, что происходит, когда можно дополнительно контролировать граничное поведение. 
Теорема 4. Пусть $\Omega \subset \mathbb{R}^{2}$ - ограниченная область с $C^{2}$-границей. Пусть $v$ - допустимое слабое решение уравнений (3) в $\Omega$ такое, что равномерно по $t$ для некоторых $\delta>0$ и $\alpha>0$ оно обладает свойством Гёлъдера с показателем $\alpha$ на множестве

$$
\Gamma_{\delta}=\{x \in \bar{\Omega}: \operatorname{dist}(x, \partial \Omega)<\delta\} .
$$

Тогда $v$ - диссипативное решение.

\section{3. Субрешения и выпуклое интегрирование}

Для доказательства теоремы 1 напомним подходы, развитые в [10], [11], внося небольшие изменения для случая областей с границей. Детали можно найти в обзоре [12] и в недавно опубликованном курсе лекций [31].

Сначала напомним определение субрешения. Зафиксируем неотрицательную функцию

$$
\bar{e} \in L^{\infty}\left(0, T ; L^{1}(\Omega)\right),
$$

которая будет играть роль плотности (кинетической) энергии. Мы будем работать в пространственно-временной области

$$
\Omega_{T}:=\Omega \times(0, T),
$$

где либо $\Omega \subset \mathbb{R}^{d}$ - открытая липшицева область, либо $\Omega=\mathbb{T}^{d}$.

ОПРЕДЕлЕниЕ 5 (субрешение). Субрешением уравнений Эйлера несжимаемой жидкости относительно плотности кинетической энергии $\bar{e}$ называется тройка

$$
(\bar{v}, \bar{u}, \bar{q}): \Omega_{T} \rightarrow \mathbb{R}^{d} \times \mathscr{S}_{0}^{d \times d} \times \mathbb{R},
$$

где $\bar{v} \in L^{\infty}(0, T ; H(\Omega)), \bar{u} \in L_{\mathrm{loc}}^{1}\left(\Omega_{T}\right), \bar{q} \in \mathscr{D}^{\prime}\left(\Omega_{T}\right)$ (т. е. $\bar{q}$ есть распределение), такая, что

$$
\left\{\begin{array}{l}
\partial_{t} \bar{v}+\operatorname{div} \bar{u}+\nabla \bar{q}=0 \\
\operatorname{div} \bar{v}=0
\end{array} \quad\right. \text { в смысле распределений }
$$

и, кроме того,

$$
\bar{v} \otimes \bar{v}-\bar{u} \leqslant \frac{2}{d} \bar{e} I \quad \text { для почти всех }(x, t) .
$$

Здесь $\mathscr{S}_{0}^{d \times d}$ - множество бесследовых симметричных матриц размера $d \times d$, a $I$ - единичная матрица. Заметим, что подрешения автоматически удовлетворяют неравенству $|\bar{v}|^{2} / 2 \leqslant \bar{e}$ п.в. Вдобавок, если п.в. в (8) имеет место равенство, то $\bar{v}$ - слабое решение уравнений Эйлера.

Неравенство (8) удобно переформулировать в терминах обобщенной nлотности энергии

$$
e(\bar{v}, \bar{u})=\frac{d}{2}|\bar{v} \otimes \bar{v}-\bar{u}|_{\infty},
$$

где $|\cdot|_{\infty}$ - операторная норма матрицы (наибольшее собственное значение в случае симметрической матрицы). Тогда неравенство (8) эквивалентно следующему:

$$
e(\bar{v}, \bar{u}) \leqslant \bar{e} \quad \text { п.в. }
$$


Ключевым моментом выпуклого интегрирования является замечание, что в случае строгого неравенства в (8) мы можем "приправить" субрешение (разумеется, множеством способов) высокочастотными колебаниями так, что получится слабое решение. Также важно заметить, что, поскольку в ходе выпуклого интегрирования к субрешению добавляются только возмущения с компактным носителем (в пространстве-времени), граничные и начальные данные получаемых слабых решений совпадают с таковыми у субрешения. В этом и заключается содержание следующей теоремы, которая по существу является предложением 2 из [11].

TЕорема 6 (критерий в терминах субрешений). Пусть $\bar{e} \in L^{\infty}\left(\Omega_{T}\right)$, u nусть $(\bar{v}, \bar{u}, \bar{q})$ - субрешение. Пусть также $\mathscr{U} \subset \Omega_{T}-$ такая подобласть, что $(\bar{v}, \bar{u}, \bar{q})$ ие непрерывны в $\mathscr{U}$,

$$
\begin{array}{ll}
e(\bar{v}, \bar{u})<\bar{e} & \text { в } \mathscr{U}, \\
e(\bar{v}, \bar{u})=\bar{e} & \text { n.в. в } \Omega_{T} \backslash \mathscr{U} .
\end{array}
$$

Тогда существует бесконечно много слабых решений уравнения Эйлера $v \in$ $L^{\infty}(0, T ; H(\Omega))$, для которых

$$
\begin{aligned}
v & =\bar{v} & & \text { п.в. в } \Omega_{T} \backslash \mathscr{U}, \\
\frac{1}{2}|v|^{2} & =\bar{e} & & \text { n.в. в } \Omega_{T}, \\
p & =\bar{q}-\frac{2}{d} \bar{e} & & \text { n.в. в } \Omega_{T} .
\end{aligned}
$$

Если к тому же

$$
\bar{v}(\cdot, t) \rightarrow v_{0}(\cdot) \quad \text { в } L^{2}(\Omega) \text { nри } t \rightarrow 0,
$$

то v являются решениями задачи Коши (3).

Упомянем также [31], где подробно обсуждается метод выпуклого интегрирования; в частности, сформулированная теорема - это теорема 7 из [31].

\section{4. Неединственность для начальных данных вращательного типа}

Используя подходы из раздела 3, здесь мы докажем теорему 1. Итак, пусть

$$
\Omega:=\left\{x \in \mathbb{R}^{2}: \rho<|x|<R\right\}
$$

- круговое кольцо, зафиксируем $r_{0} \in(\rho, R)$ и положим

$$
v_{0}(x)= \begin{cases}-\frac{1}{|x|^{3}} x^{\perp}, & |x|<r_{0}, \\ \frac{1}{|x|^{3}} x^{\perp}, & |x|>r_{0},\end{cases}
$$

где $x^{\perp}=\left(\begin{array}{c}x_{2} \\ -x_{1}\end{array}\right)$. Мы построим субрешения методом, аналогичным методу работы [30]. 
В силу теоремы 6 из предыдущего раздела, достаточно проверить существование субрешений с нужными свойствами. Зафиксируем малые константы $\lambda>0$ ("скорость распространения турбулентности") и $\varepsilon \geqslant 0$ ("интенсивность диссипации энергии"), значения которых мы уточним в дальнейшем.

Мы будем искать субрешения $(\bar{v}, \bar{u}, \bar{q})$ (cp. с определением 5 - мы еще не зафиксировали функцию плотности энергии $\bar{e})$ вида

$$
\bar{v}(x, t)=\alpha(r, t)\left(\begin{array}{c}
\sin \theta \\
-\cos \theta
\end{array}\right),
$$

где $\alpha(r, 0)=\alpha_{0}(r),(r, \theta)$ - полярные координаты в $\mathbb{R}^{2}$,

$$
\begin{aligned}
\bar{u}(x, t) & =\left(\begin{array}{cc}
\cos \theta & \sin \theta \\
\sin \theta & -\cos \theta
\end{array}\right)\left(\begin{array}{cc}
\beta(r, t) & \gamma(r, t) \\
\gamma(r, t) & -\beta(r, t)
\end{array}\right)\left(\begin{array}{cc}
\cos \theta & \sin \theta \\
\sin \theta & -\cos \theta
\end{array}\right) \\
& =\left(\begin{array}{cc}
\beta \cos (2 \theta)+\gamma \sin (2 \theta) & \beta \sin (2 \theta)-\gamma \cos (2 \theta) \\
\beta \sin (2 \theta)-\gamma \cos (2 \theta) & -\beta \cos (2 \theta)-\gamma \sin (2 \theta)
\end{array}\right)
\end{aligned}
$$

и

$$
\bar{q}=\bar{q}(r)
$$

Заметим, кстати, что, выбрав $\alpha(r, t)=\alpha_{0}(r)$ для всех $t \geqslant 0, \beta=-\alpha^{2} / 2$, $\gamma=0$ и

$$
\bar{q}(r)=\frac{1}{2} \alpha^{2}+\int_{\rho}^{r} \frac{\alpha(s)^{2}}{s} d s,
$$

мы получаем хорошо известное стационарное решение (в формуле для $\bar{q}$ интеграл выражает физическое давление).

Подставив этот анзац в (7), мы получим два уравнения. Точнее, используя формулы

$$
\nabla_{x} r=\left(\begin{array}{c}
\cos \theta \\
\sin \theta
\end{array}\right) \quad \text { и } \quad \nabla_{x} \theta=\frac{1}{r}\left(\begin{array}{c}
-\sin \theta \\
\cos \theta
\end{array}\right) \text {, }
$$

мы получаем

$$
\begin{aligned}
\partial_{t} \alpha \sin \theta & +\partial_{r} \beta[\cos \theta \cos (2 \theta)+\sin \theta \sin (2 \theta)]+\partial_{r} \gamma[\cos \theta \sin (2 \theta)-\sin \theta \cos (2 \theta)] \\
& +\frac{2}{r} \beta[\sin \theta \sin (2 \theta)+\cos \theta \cos (2 \theta)]+\frac{2}{r} \gamma[-\sin \theta \cos (2 \theta)+\cos \theta \sin (2 \theta)] \\
& +\partial_{r} \bar{q} \cos \theta=0
\end{aligned}
$$

И

$$
\begin{aligned}
-\partial_{t} \alpha \cos \theta & +\partial_{r} \beta[\cos \theta \sin (2 \theta)-\sin \theta \cos (2 \theta)]+\partial_{r} \gamma[-\cos \theta \cos (2 \theta)-\sin \theta \sin (2 \theta)] \\
& +\frac{2}{r} \beta[-\sin \theta \cos (2 \theta)+\cos \theta \sin (2 \theta)]+\frac{2}{r} \gamma[-\sin \theta \sin (2 \theta)-\cos \theta \cos (2 \theta)] \\
& +\partial_{r} \bar{q} \sin \theta=0 .
\end{aligned}
$$

Если первое уравнение умножить на $\sin \theta$, сложить его со вторым, умноженным на $\cos \theta$, воспользоваться тождествами $\cos ^{2} \theta-\sin ^{2} \theta=\cos (2 \theta)$ и $2 \sin \theta \cos \theta=$ 
$\sin (2 \theta)$, а затем выделить члены, содержащие $\sin (2 \theta)$ и $\cos (2 \theta)$, то мы придем к двум уравнениям

$$
\begin{aligned}
& \partial_{r} \beta+\frac{2}{r} \beta+\partial_{r} \bar{q}=0, \\
& \partial_{t} \alpha+\partial_{r} \gamma+\frac{2}{r} \gamma=0 .
\end{aligned}
$$

Легко проверить, что для выбранного анзаца они эквивалентны исходной системе (7).

Если взять $\bar{q}(r)$ вида (14) и $\beta=-\alpha^{2} / 2$, то первое уравнение выполнено, совершенно (с точностью до знака) аналогично работе [30]. Второе уравнение также похоже на уравнение в [30], но оно содержит дополнительный "центробежный" член $(2 / r) \gamma$ и мы не можем просто положить $\gamma=\alpha^{2} / 2$, как в [30], и получить уравнение Бюргерса. Однако мы замечаем, что $\partial_{r}\left(r^{2} \gamma\right)=2 r \gamma+r^{2} \partial_{r} \gamma$, и полагаем

$$
\alpha(r, t)=\frac{1}{r^{2}} f(r, t)
$$

и

$$
\gamma=-\frac{\lambda}{2 r^{2}}\left(1-f^{2}\right)=-\frac{\lambda}{2}\left(\frac{1}{r^{2}}-r^{2} \alpha^{2}\right),
$$

так что второе уравнение в (15), после умножения на $r^{2}$, превращается в уравнение Бюргерса

$$
\partial_{t} f+\frac{\lambda}{2} \partial_{r}\left(f^{2}\right)=0 .
$$

Теперь начальным данным (6) для $\alpha$ соответствуют начальные данные

$$
f(r, 0)= \begin{cases}-1 & \text { при } \rho<r<r_{0}, \\ 1 & \text { при } r_{0}<r<R .\end{cases}
$$

Уравнение Бюргерса (17) с этими начальными данными имеет решение в виде волны разрежения для $t \in[0, T]$, при условии, что $\lambda>0$ достаточно мало (при выбранных $T$ и $\left.r_{0}, \rho<r_{0}<R\right)$; оно задается равенствами

$$
f(r, t)= \begin{cases}-1 & \text { при } \rho<r<r_{0}-\lambda t, \\ \frac{r-r_{0}}{\lambda t} & \text { при } r_{0}-\lambda t<r<r_{0}+\lambda t, \\ 1 & \text { при } r_{0}+\lambda t<r<R .\end{cases}
$$

Итак, полагая $\alpha(r, t)=f(r, t) / r^{2}$, для $f$ из $(18), \beta=-\alpha^{2} / 2, \gamma$ из (16) и $\bar{q}$ из (14) мы находим решение уравнений (7) с начальными данными, соответствующими (12).

Остается разобраться с обобщенной энергией. Поскольку $\bar{u}$ задано в (13) и вдобавок

$$
\begin{aligned}
\bar{v} \otimes \bar{v} & =\alpha(r, t)^{2}\left(\begin{array}{cc}
\cos ^{2} \theta & -\sin \theta \cos \theta \\
-\sin \theta \cos \theta & \cos ^{2} \theta
\end{array}\right) \\
& =\left(\begin{array}{cc}
\cos \theta & \sin \theta \\
\sin \theta & -\cos \theta
\end{array}\right)\left(\begin{array}{cc}
0 & 0 \\
0 & \alpha(r, t)^{2}
\end{array}\right)\left(\begin{array}{cc}
\cos \theta & \sin \theta \\
\sin \theta & -\cos \theta
\end{array}\right),
\end{aligned}
$$


и поскольку собственные значения матрицы не меняются при сопряжении с помощью ортогонального преобразования, можно вычислить $e(\bar{v}, \bar{u})=|\bar{v} \otimes \bar{v}-\bar{u}|_{\infty}$, найдя наибольшее собственное значение матрицы

$$
\left(\begin{array}{cc}
-\beta & -\gamma \\
-\gamma & \alpha^{2}+\beta
\end{array}\right)=\left(\begin{array}{cc}
\frac{1}{2} \alpha^{2} & \frac{\lambda}{2}\left(\frac{1}{r^{2}}-r^{2} \alpha^{2}\right) \\
\frac{\lambda}{2}\left(\frac{1}{r^{2}}-r^{2} \alpha^{2}\right) & \frac{1}{2} \alpha^{2}
\end{array}\right)
$$

Учитывая, что $|\alpha| \leqslant 1 / r^{2}$ и $\lambda \geqslant 0$, легко видеть, что

$$
e(\bar{v}, \bar{u})=\frac{1}{2} \alpha^{2}+\frac{\lambda}{2}\left(\frac{1}{r^{2}}-r^{2} \alpha^{2}\right)=\frac{1}{2 r^{4}}\left[1-\left(1-r^{2} \lambda\right)\left(1-f(r, t)^{2}\right)\right] .
$$

Наконец, положим

$$
\bar{e}(r, t)=\frac{1}{2 r^{4}}\left[1-\varepsilon\left(1-r^{2} \lambda\right)\left(1-f(r, t)^{2}\right)\right],
$$

где $\varepsilon$ достаточно мало, так что $\bar{e}>0$. Заметим, что

$$
e(\bar{v}, \bar{u}) \leqslant \bar{e} \leqslant \frac{1}{2}\left|v_{0}\right|^{2} \quad \text { в } \Omega_{T} .
$$

Точнее, подводя итог выкладкам этого раздела, мы получаем следующий результат.

ПРЕДЛОЖЕНИЕ 7. Для любого выбора постоянных $\varepsilon, \lambda$, удовлетворяющих условиям

$$
\begin{aligned}
& 0<\lambda<\min \left\{\frac{1}{R^{2}}, \frac{r_{0}-\rho}{T}, \frac{R-r_{0}}{T}\right\}, \\
& 0 \leqslant \varepsilon<\frac{1}{1-\rho^{2} \lambda},
\end{aligned}
$$

в $\Omega_{T}$ существует такое субрешение $(\bar{v}, \bar{u}, \bar{q})$ относительно плотности кинетической энергии

$$
\bar{e}(r, t)=\frac{1}{2 r^{4}}\left[1-\varepsilon\left(1-r^{2} \lambda\right)\left(1-f(r, t)^{2}\right)\right]
$$

с начальными даннъми $\bar{v}(x, 0)=v_{0}(x)$ из (12), что для

$$
\mathscr{U}:=\left\{x \in \mathbb{R}^{2}: r_{0}-\lambda t<|x|<r_{0}+\lambda t\right\}
$$

выполнены соотношения

$$
\begin{array}{ll}
e(\bar{v}, \bar{u})<\bar{e} & \text { в } \mathscr{U}, \\
e(\bar{v}, \bar{u})=\bar{e} & \text { в } \Omega_{T} \backslash \mathscr{U} .
\end{array}
$$

Теперь мы можем доказать теорему 1. 
ДоказАТЕЛЬСтво теоремы 1. Применив предложение 7 с $\varepsilon \geqslant 0$, получим субрешение $(\bar{v}, \bar{u}, \bar{q})$. Воспользовавшись результатом теоремы 6 для этого субрешения, мы видим, что существует бесконечно много слабых решений $v \in L^{\infty}(0, T ; H(\Omega))$, удовлетворяющих равенству $|v|^{2}=2 \bar{e}$ п.в. в $\Omega_{T}$ и с начальными данными $v_{0}$. Для проверки их допустимости заметим, что

$$
\int_{\Omega}|v(x, t)|^{2} d x=\int_{\Omega} 2 \bar{e}(x, t) d x \leqslant \int_{\Omega} \frac{1}{|x|^{4}} d x=\int_{\Omega}\left|v_{0}(x)\right|^{2} d x .
$$

Наконец, отметим, что, выбрав $\varepsilon>0$, мы получим решения со строго убывающей энергией, а выбрав $\varepsilon=0$, мы получим сохранение энергии. Теорема доказана.

\section{5. Единственность предела нулевой вязкости}

ДокАЗАТЕЛЬСтво ПРЕДЛОжЕНИЯ 2. Рассмотрим уравнения Навье-Стокса с вязкостью $\varepsilon>0$ :

$$
\begin{aligned}
\partial_{t} v_{\varepsilon}+v_{\varepsilon} \cdot \nabla v_{\varepsilon}+\nabla p_{\varepsilon} & =\varepsilon \Delta v_{\varepsilon}, \\
\operatorname{div} v_{\varepsilon} & =0, \\
v_{\varepsilon}(\cdot, 0) & =v_{0}, \\
\left.v_{\varepsilon}\right|_{\partial \Omega} & =0 .
\end{aligned}
$$

Как известно, в случае двух пространственных переменных уравнения Навье-Стокса имеют единственное слабое решение (решение Лере-Хопфа), удовлетворяющее энергетическому равенству

$$
\frac{1}{2} \int_{\Omega}\left|v_{\varepsilon}(x, t)\right|^{2} d x+\varepsilon \int_{0}^{t} \int_{\Omega}\left|\nabla v_{\varepsilon}(x, s)\right|^{2} d x d s=\frac{1}{2} \int_{\Omega}\left|v_{0}(x)\right|^{2} d x
$$

для всякого $t \in[0, T]$; см. подробнее, к примеру, [17]. Оказывается, что, когда начальные данные $v_{0}$ обладают вращательной симметрией вида (4), единственное решение Лере-Хопфа также обладает такой симметрией.

Для доказательства воспользуемся анзацем

$$
v_{\varepsilon}(x, t)=\alpha_{\varepsilon}(r, t)\left(\begin{array}{c}
\sin \theta \\
-\cos \theta
\end{array}\right)
$$

$p_{\varepsilon}=p_{\varepsilon}(r)$, где мы опять используем полярные координаты. Подставив этот анзац в первое уравнение из (20), получим

$$
\partial_{t} \alpha_{\varepsilon} \sin \theta-\frac{\alpha_{\varepsilon}^{2}}{r} \cos \theta+\partial_{r} p_{\varepsilon} \cos \theta=\varepsilon\left(\frac{\partial_{r} \alpha_{\varepsilon}}{r}+\partial_{r}^{2} \alpha_{\varepsilon}-\frac{\alpha_{\varepsilon}}{r^{2}}\right) \sin \theta
$$

Взяв

$$
p_{\varepsilon}(r)=\int_{\rho}^{r} \frac{\alpha_{\varepsilon}(s)^{2}}{s} d s
$$

и поделив на $\sin \theta$, мы придем к параболическому уравнению

$$
\partial_{t} \alpha_{\varepsilon}=\varepsilon\left(\frac{\partial_{r} \alpha_{\varepsilon}}{r}+\partial_{r}^{2} \alpha_{\varepsilon}-\frac{\alpha_{\varepsilon}}{r^{2}}\right)
$$


Подставив анзац во второе уравнение из (20), мы также получим (22), что легко увидеть после аналогичных выкладок. Более того, бездивергентность вытекает автоматически, начальное условие превращается в

$$
\alpha_{\varepsilon}(\cdot, 0)=\alpha_{0}
$$

где $\alpha_{0}$ задано в (6), а граничное условие переходит в

$$
\alpha_{\varepsilon}(\rho)=\alpha_{\varepsilon}(R)=0
$$

Итак, мы приходим к корректной начально-краевой задаче (22)-(24). Хорошо известно (см., к примеру, [14; раздел 7.1]), что для всякого $\varepsilon>0$ это параболическое уравнение имеет единственное слабое решение. Однако из проведенных выкладок следует, что если $\alpha_{\varepsilon}$ - решение параболической задачи, то соответствующее $v_{\varepsilon}$, заданное в $(21)$, является (единственным) решением Лере-Хопфа задачи Навье-Стокса (20) и в то же время решает начально-краевую задачу для уравнения теплопроводности

$$
\begin{aligned}
\partial_{t} v_{\varepsilon} & =\varepsilon \Delta v_{\varepsilon}, \\
\operatorname{div} v_{\varepsilon} & =0, \\
v_{\varepsilon}(\cdot, 0) & =v_{0}, \\
\left.v_{\varepsilon}\right|_{\partial \Omega} & =0 .
\end{aligned}
$$

Поскольку решения уравнения теплопроводности сходятся в сильном смысле к стационарному решению и поскольку мы показали, что для наших начальных данных уравнение теплопроводности совпадает с уравнениями Навье-Стокса, предложение доказано.

ЗАмЕЧАниЕ 8. Изложенные выше рассуждения можно провести и для начальных данных, заданных в цилиндре $Z=\Omega \times \mathbb{T} \subset \mathbb{R}^{2} \times \mathbb{T}$, где, как и прежде, $\Omega \subset \mathbb{R}^{2}$ - кольцо. Действительно, для так называемых $21 / 2$-мерных начальных данных $V_{0}\left(x_{1}, x_{2}\right)=\left(v_{0}\left(x_{1}, x_{2}\right), w\left(x_{1}, x_{2}\right)\right)$ на $Z$, где $v_{0}$ имеет вид $(4)$, имеется бесконечно много допустимых слабых решений, но только решение

$$
V\left(x_{1}, x_{2}, t\right)=\left(v_{0}\left(x_{1}, x_{2}\right), w\left(x_{1}-\left(v_{0}\right)_{1} t, x_{2}-\left(v_{0}\right)_{2} t\right)\right)
$$

возникает как предел нулевой вязкости. Мы опускаем подробности, но отметим, что это можно доказать аналогично [4], где такой анализ был проведен для сдвиговых течений.

\section{6. Диссипативные решения}

Пусть $S(w)=\left(\nabla w+\nabla w^{t}\right) / 2$ обозначает симметризованный градиент векторного поля $w$, и пусть

$$
E(w)=-\partial_{t} w-P(w \cdot \nabla w)
$$

где $P$ - проектор Лере-Гельмгольца на $H(\Omega)$. 
Следующее определение из [22] приведено здесь в версии из [2] для ограниченных областей. Читатель может обратиться к этим источникам за мотивировками определения.

ОПРеДЕЛЕНиЕ 9. Пусть $\Omega$ - ограниченная область с $C^{1}$-границей. Векторное поле $v \in C\left([0, T] ; H_{\mathrm{w}}(\Omega)\right)$ называется диссипативным решением уравнений Эйлера (3), если для всякого бездивергентного основного векторного поля $w \in C^{1}(\bar{\Omega} \times[0, T])$, удовлетворяющего условию $w \cdot \nu \uparrow$ Ґа $=0$, выполнено неравенство

$$
\begin{aligned}
\int_{\Omega}|v-w|^{2} d x \leqslant & \exp \left(2 \int_{0}^{t}\|S(w)\|_{\infty} d s\right) \int_{\Omega}|v(x, 0)-w(x, 0)|^{2} d x \\
& +2 \int_{0}^{t} \int_{\Omega} \exp \left(2 \int_{s}^{t}\|S(w)\|_{\infty} d \tau\right) E(w) \cdot(v-w) d x d s
\end{aligned}
$$

для всех $t \in[0, T]$.

Из этого определения сразу вытекает единственность сильных решений в классе слабых (предложение 4.1 из [22]).

ПреДЛОЖенИЕ 10. Пусть $v \in C^{1}(\bar{\Omega} \times[0, T])$ - решение уравнений Эйлера (3). Тогда $v$-единственнъй представитель класса диссипативных решений с теми же начальными данными.

Это можно увидеть, просто выбрав $w=v$ в качестве основной функции в определении диссипативных решений.

Докажем теперь следствие 3, показав, что допустимые решения в ограниченных областях не обязательно единственны, даже в случае гладких начальных данных.

ДоКАЗАТЕЛЬСТВо СЛЕДСТВИЯ 3. Вспоминая конструкцию из раздела 4, положим

$$
\widetilde{\Omega}=\left\{x \in \mathbb{R}^{2}: \rho<|x|<r_{0}\right\} \subset \Omega .
$$

Из определения субрешения непосредственно следует, что его ограничение на подобласть само является субрешением. Так что мы можем рассматривать субрешение $(\bar{v}, \bar{u}, \bar{q})$ из раздела 4 как субрешение в $\widetilde{\Omega}$ с той же плотностью энергии $\bar{e}$, что и в предложении 7 , и с начальными данными

$$
\bar{v}(x, 0)=-\frac{x^{\perp}}{|x|^{3}} \quad \text { при } x \in \widetilde{\Omega}
$$

(ср. с (12)). Теперь мы применим к этому субрешению в $\widetilde{\Omega}$ теорему 6 , что, как и в доказательстве теоремы 1 , даст нам бесконечно много допустимых слабых решений.

Поскольку начальные данные $\bar{v}(x, 0)$ в $\widetilde{\Omega}$ гладкие, имеется единственное сильное решение (на самом деле равное стационарному решению). Таким образом, единственность сильных решений в классе слабых не имеет места, что с необходимостью означает, что нестационарные допустимые слабые решения не диссипативны по Лионсу. 


\section{7. Критерий диссипативности допустимого решения}

Как мы видели, в ограниченных областях допустимые слабые решения не обязательно диссипативны. Однако если такое решение обладает свойством Гёльдера вблизи границы области, то сформулированная выше теорема 4 утверждает, что ситуация меняется. В этом разделе мы собираемся доказать эту теорему. Мы следуем дополнению В к [11], но должны учитывать при этом, что в определении диссипативных решений нам придется работать с основными функциями, не обязательно имеющими компактный носитель в $\Omega$.

Итак, пусть $\Omega$ - ограниченная область с $C^{2}$-границей в $\mathbb{R}^{2}$, а $v$ - допустимое слабое решение уравнений Эйлера (3), как в формулировке теоремы 4. Будем пока считать, что для всякого бездивергентного поля $w \in C^{1}(\bar{\Omega} \times[0, T])$, удовлетворяющего краевому условию, выполнено равенство

$$
\frac{d}{d t} \int_{\Omega} v \cdot w d x=\int_{\Omega}(S(w)(v-w) \cdot(v-w)-E(w) \cdot v) d x
$$

в смысле обобщенных функций, где $E(w)$ - величина, определенная в начале раздела 6. Мы покажем, что из (26) уже следует, что $v$ - диссипативное решение. Действительно, это доказывается в точности так же, как в [11]: с одной стороны, поскольку $v$ допустимое,

$$
\frac{d}{d t} \int_{\Omega}|v|^{2} d x \leqslant 0
$$

в смысле обобщенных функций. С другой стороны, используя определение $E(w)$ и тождество $\int_{\Omega}(w \cdot \nabla w) \cdot w d x=0$ (следующее из $\left.w \cdot \nu \uparrow д \Omega=0\right)$, получаем

$$
\frac{d}{d t} \int_{\Omega}|w|^{2} d x=-2 \int_{\Omega} E(w) \cdot w d x
$$

Taк как

$$
\int_{\Omega}|v-w|^{2} d x=\int_{\Omega}|v|^{2} d x+\int_{\Omega}|w|^{2} d x-2 \int_{\Omega} v \cdot w d x,
$$

то, объединяя это равенство с (26), (27) и (28), мы заключаем, что

$$
\begin{aligned}
\frac{d}{d t} \int_{\Omega}|v-w|^{2} d x & \leqslant 2 \int_{\Omega}(E(w) \cdot(v-w)-S(w)(v-w) \cdot(v-w)) d x \\
& \leqslant 2 \int_{\Omega} E(w) \cdot(v-w) d x+2\|S(w)\|_{\infty} \int_{\Omega}|v-w|^{2} d x
\end{aligned}
$$

в смысле обобщенных функций. Теперь, как в [11], мы применим неравенство Гронуолла и получим (25) для всех $t \in[0, T]$. Таким образом, остается доказать (26) для любой основной функции $w$.

В [11] тождество (26) было доказано для $w$, имеющей компактный носитель в $\Omega$ почти во все моменты времени (см. рассуждения, следующие за (96) в [11]). Теперь допустим, что $w \in C^{1}(\bar{\Omega} \times[0, T])$ - бездивергентное векторное поле, удовлетворяющее $\left.w \cdot \nu\right|_{\partial \Omega}=0$, но не обязательно имеющее компактный носитель в пространстве. Мы аппроксимируем $w$ подходящими векторными 
полями с компактным носителем в духе Като [21] (см., в частности, [21; раздел 4]).

Для начала предположим, что область $\Omega$ односвязна, так что у $\partial \Omega$ одна компонента связности. Поскольку поле $w$ бездивергентно, существует функция $\psi \in C\left([0, T] ; C^{2}(\bar{\Omega})\right) \cap C^{1}(\bar{\Omega} \times[0, T])$, для которой

$$
w(x, t)=\nabla^{\perp} \psi(x, t)
$$

и $\psi \uparrow_{\text {да }}=0$. Теперь пусть $\chi:[0, \infty) \rightarrow \mathbb{R}-$ такая гладкая неотрицательная функция, что

$$
\chi(s)= \begin{cases}0 & \text { при } s<1 \\ 1 & \text { при } s>2\end{cases}
$$

положим

$$
w_{\varepsilon}(x, t)=\nabla^{\perp}\left(\chi\left(\frac{\operatorname{dist}(x, \partial \Omega)}{\varepsilon}\right) \psi(x, t)\right) .
$$

Тогда по лемме 14.16 из [18] для некоторого $\eta>0$, зависящего от $\Omega$, функция $x \mapsto \operatorname{dist}(x, \partial \Omega)$ является $C^{2}$-гладкой в области

$$
\Gamma_{\eta}=\{x \in \bar{\Omega}: \operatorname{dist}(x, \partial \Omega)<\eta\}
$$

так что $w_{\varepsilon} \in C_{c}^{1}(\Omega \times[0, T])$ для достаточно малых $\varepsilon>0$. Значит, для $w_{\varepsilon}$ выполнено равенство (26):

$$
\frac{d}{d t} \int_{\Omega} v \cdot w_{\varepsilon} d x=\int_{\Omega}\left(S\left(w_{\varepsilon}\right)\left(v-w_{\varepsilon}\right) \cdot\left(v-w_{\varepsilon}\right)-E\left(w_{\varepsilon}\right) \cdot v\right) d x .
$$

Теперь для доказательства (26) устремим $\varepsilon$ к нулю.

Обозначив $d(x)=\operatorname{dist}(x, \partial \Omega)$, из определения $w_{\varepsilon}$ получим

$$
w_{\varepsilon}=\chi\left(\frac{d}{\varepsilon}\right) \nabla^{\perp} \psi+\frac{1}{\varepsilon} \chi^{\prime}\left(\frac{d}{\varepsilon}\right) \psi \nabla^{\perp} d,
$$

и поскольку $\psi \in C\left([0, T] ; C^{2}(\bar{\Omega})\right)$ и $\psi\lceil\partial \Omega=0$, существует константа $C$, не зависящая от $t$ и $\varepsilon$, для которой

$$
|\psi(x, t)| \leqslant C d(x)
$$

при всех $x \in \bar{\Omega}$. Кроме того, поскольку $\chi^{\prime}(\cdot / \varepsilon)$ имеет носитель в $(\varepsilon, 2 \varepsilon)$, а $|\nabla d| \leqslant 1$, из (31) следует сходимость

$$
w_{\varepsilon} \rightarrow w \quad \text { в } L^{\infty}\left([0, T] ; L^{2}(\Omega)\right)
$$

в сильном смысле при $\varepsilon \rightarrow 0$. Для левой части (30) отсюда немедленно вытекает, что

$$
\frac{d}{d t} \int_{\Omega} v \cdot w_{\varepsilon} d x \rightarrow \frac{d}{d t} \int_{\Omega} v \cdot w d x
$$


в смысле обобщенных функций. Вдобавок, используя определение $E\left(w_{\varepsilon}\right)$, мы можем переписать (30) в виде

$$
\begin{aligned}
& \int_{\Omega}(\left.S\left(w_{\varepsilon}\right)\left(v-w_{\varepsilon}\right) \cdot\left(v-w_{\varepsilon}\right)-E\left(w_{\varepsilon}\right) \cdot v\right) d x \\
& \quad=\int_{\Omega}\left[\partial_{t} w_{\varepsilon} \cdot v+\left(v \cdot \nabla w_{\varepsilon}\right) \cdot v-\left(\left(v-w_{\varepsilon}\right) \cdot \nabla w_{\varepsilon}\right) \cdot w_{\varepsilon}\right] d x,
\end{aligned}
$$

и правая часть (26) допускает аналогичное выражение.

Заметим теперь, что, опять-таки в силу (32),

$$
\int_{\Omega} \partial_{t} w_{\varepsilon} \cdot v d x \rightarrow \int_{\Omega} \partial_{t} w \cdot v d x
$$

в смысле обобщенных функций и что

$$
\int_{\Omega}\left(\left(v-w_{\varepsilon}\right) \cdot \nabla w_{\varepsilon}\right) \cdot w_{\varepsilon} d x=\int_{\Omega}((v-w) \cdot \nabla w) \cdot w d x=0
$$

ввиду формулы $((v-w) \cdot \nabla w) \cdot w=(v-w) \cdot(1 / 2) \nabla|w|^{2}$ и включения $v-w \in H(\Omega)$ (и аналогичных соображений относительно $\left.\left(\left(v-w_{\varepsilon}\right) \cdot \nabla w_{\varepsilon}\right) \cdot w_{\varepsilon}\right)$.

Для завершения доказательства (26) и теоремы 4 в целом остается показать, что

$$
\int_{\Omega}\left(v \cdot \nabla w_{\varepsilon}\right) \cdot v d x \rightarrow \int_{\Omega}(v \cdot \nabla w) \cdot v d x
$$

в смысле обобщенных функций при $\varepsilon \rightarrow 0$.

С этой целью заметим, что у точек $x \in \Omega$, достаточно близких к $\partial \Omega$, существует единственная ближайшая точка $\widehat{x} \in \partial \Omega$, так что

$$
x=\widehat{x}+d(x) \nu(\widehat{x}) .
$$

Через $\tau(\widehat{x})=\left(-\nu_{2}(\widehat{x}), \nu_{1}(\widehat{x})\right)$ обозначим единичный вектор, касательный к $\partial \Omega$ в $\widehat{x}$, и введем обозначения $v_{\tau}(x)=v(x) \cdot \tau(\widehat{x}), \partial_{\tau} w_{\nu}(x)=\nabla w_{\nu}(x) \cdot \tau(\widehat{x})$ и т. п. (напомним, что $\widehat{x}$ однозначно определяется по $x$ ). Для достаточно малых $\varepsilon$ выполнено равенство (вспомним (29))

$$
\begin{aligned}
\int_{\Omega}\left(v \cdot \nabla\left(w_{\varepsilon}-w\right)\right) \cdot v d x= & \int_{\Gamma_{2 \varepsilon}} v_{\nu} \partial_{\nu}\left(w_{\varepsilon}-w\right)_{\nu} v_{\nu} d x+\int_{\Gamma_{2 \varepsilon}} v_{\nu} \partial_{\nu}\left(w_{\varepsilon}-w\right)_{\tau} v_{\tau} d x \\
& +\int_{\Gamma_{2 \varepsilon}} v_{\tau} \partial_{\tau}\left(w_{\varepsilon}-w\right)_{\nu} v_{\nu} d x+\int_{\Gamma_{2 \varepsilon}} v_{\tau} \partial_{\tau}\left(w_{\varepsilon}-w\right)_{\tau} v_{\tau} d x \\
= & : I_{1}+I_{2}+I_{3}+I_{4} .
\end{aligned}
$$

Используя (31) и равенство $\nabla^{\perp} \psi=w$, а также замечая, что $\nabla d=\nu$, найдем

$$
\begin{aligned}
\left(w_{\varepsilon}-w\right)_{\nu} & =\left(\chi\left(\frac{d}{\varepsilon}\right)-1\right) \partial_{\tau} \psi=\left(\chi\left(\frac{d}{\varepsilon}\right)-1\right) w_{\nu}, \\
\left(w_{\varepsilon}-w\right)_{\tau} & =-\left(\chi\left(\frac{d}{\varepsilon}\right)-1\right) \partial_{\nu} \psi+\frac{1}{\varepsilon} \chi^{\prime}\left(\frac{d}{\varepsilon}\right) \psi \\
& =\left(\chi\left(\frac{d}{\varepsilon}\right)-1\right) w_{\tau}+\frac{1}{\varepsilon} \chi^{\prime}\left(\frac{d}{\varepsilon}\right) \psi,
\end{aligned}
$$




$$
\begin{aligned}
& \partial_{\nu}\left(w_{\varepsilon}-w\right)_{\nu}=\frac{1}{\varepsilon} \chi^{\prime}\left(\frac{d}{\varepsilon}\right) w_{\nu}+\left(\chi\left(\frac{d}{\varepsilon}\right)-1\right) \partial_{\nu} w_{\nu}, \\
& \partial_{\nu}\left(w_{\varepsilon}-w\right)_{\tau}=\left(\chi\left(\frac{d}{\varepsilon}\right)-1\right) \partial_{\nu} w_{\tau}+\frac{1}{\varepsilon^{2}} \chi^{\prime \prime}\left(\frac{d}{\varepsilon}\right) \psi, \\
& \partial_{\tau}\left(w_{\varepsilon}-w\right)_{\nu}=\left(\chi\left(\frac{d}{\varepsilon}\right)-1\right) \partial_{\tau} w_{\nu}, \\
& \partial_{\tau}\left(w_{\varepsilon}-w\right)_{\tau}=\left(\chi\left(\frac{d}{\varepsilon}\right)-1\right) \partial_{\tau} w_{\tau}+\frac{1}{\varepsilon} \chi^{\prime}\left(\frac{1}{\varepsilon}\right) w_{\nu} .
\end{aligned}
$$

Прежде чем оценить $I_{1}-I_{4}$ с помощью (34)-(37), отметим еще несколько фактов. Как мы уже указывали, $|\psi(x)| \leqslant C d(x)$ для некоторой константы $C$, не зависящей от $t$. Далее, поскольку $w \in C^{1}(\bar{\Omega} \times[0, T])$ и $w_{\nu}=0$ на $\partial \Omega$, аналогично можно найти константу, не зависящую от $t$, с которой $\left|w_{\nu}(x)\right| \leqslant C d(x)$. По предположению, при достаточно малом $\varepsilon$ функция $v$ равномерно (по $t$ ) гёльдерова в $\Gamma_{2 \varepsilon}$ с показателем $\alpha$, а так как из включения $v \in H(\Omega)$ следует, что $v_{\nu}=0$ на $\partial \Omega$ (ср. с [16; гл. III]), мы получаем еще одну константу, не зависящую от времени, с которой $\left|v_{\nu}(x)\right| \leqslant C d(x)^{\alpha}$ в $\Gamma_{2 \varepsilon}$. Заметим, наконец, что $\psi$, $w$ и $v$ равномерно ограничены на $\Gamma_{2 \varepsilon}$, если только $\varepsilon$ мало, и что с некоторой константой, не зависящей от $\varepsilon$, верно неравенство $\left|\Gamma_{2 \varepsilon}\right| \leqslant C \varepsilon$.

В свете этих фактов мы можем использовать (34)-(37), чтобы получить следующие равномерные по времени оценки:

$$
\begin{aligned}
\left|I_{1}\right| & \leqslant \frac{1}{\varepsilon} \int_{\Gamma_{2 \varepsilon}} v_{\nu}^{2}\left\|\chi^{\prime}\right\|_{\infty}\left\|w_{\tau}\right\|_{\infty} d x+\int_{\Gamma_{2 \varepsilon}} v_{\nu}^{2}\|\chi-1\|_{\infty}\left\|\partial_{\nu} w_{\nu}\right\|_{\infty} d x \\
& \leqslant C \varepsilon^{2 \alpha+1}+C \varepsilon^{2 \alpha+1} \rightarrow 0, \\
\left|I_{2}\right| & \leqslant \int_{\Gamma_{2 \varepsilon}}\left|v_{\nu}\right|\left|v_{\tau}\right|\|\chi-1\|_{\infty}\left\|\partial_{\nu} w_{\tau}\right\|_{\infty} d x+\frac{1}{\varepsilon^{2}} \int_{\Gamma_{2 \varepsilon}}\left|v_{\nu}\right|\left|v_{\tau}\right|\left\|\chi^{\prime \prime}\right\|_{\infty}|\psi| d x \\
& \leqslant C \varepsilon^{\alpha+1}+C \varepsilon^{\alpha} \rightarrow 0, \\
\left|I_{3}\right| & \leqslant \int_{\Gamma_{2 \varepsilon}}\left|v_{\nu}\right|\left|v_{\tau}\right|\|\chi-1\|_{\infty}\left\|\partial_{\tau} w_{\nu}\right\|_{\infty} d x \leqslant C \varepsilon^{\alpha+1} \rightarrow 0, \\
\left|I_{4}\right| & \leqslant \int_{\Gamma_{2 \varepsilon}} v_{\tau}^{2}\|\chi-1\|_{\infty}\left\|\partial_{\tau} w_{\tau}\right\|_{\infty} d x+\frac{1}{\varepsilon} \int_{\Gamma_{2 \varepsilon}} v_{\tau}^{2}\left\|\chi^{\prime}\right\|_{\infty}\left|w_{\nu}\right| \\
& \leqslant C \varepsilon+C \varepsilon \rightarrow 0 .
\end{aligned}
$$

Это доказывает теорему 4 в односвязной области $\Omega$.

На заключительном шаге мы убедимся, что доказательство легко подправить в духе раздела 1.4 работы [20] для общего случая, когда $\partial \Omega$ состоит из $N$ компонент $\Gamma^{1}, \ldots, \Gamma^{N}$. По-прежнему, мы можем найти $\psi \in C\left([0, T] ; C^{2}(\bar{\Omega})\right) \cap$ $C^{1}(\bar{\Omega} \times[0, T])$, для которого $\nabla^{\perp} \psi=w$, но теперь мы не можем требовать, чтобы $\psi\left\lceil\partial \Omega=0\right.$. Вместо этого $\psi$ теперь принимает постоянное значение $\psi^{i}$ на каждой $\Gamma^{i}$, но числа $\psi^{i}$ могут быть разными. Если $\varepsilon>0$ достаточно мало, то множества

$$
\Gamma_{2 \varepsilon}^{i}=\left\{x \in \bar{\Omega}: \operatorname{dist}\left(x, \Gamma^{i}\right)<2 \varepsilon\right\}, \quad i=1, \ldots, N
$$


не пересекаются, и, полагая

$$
w_{\varepsilon}(x)= \begin{cases}\nabla^{\perp}\left(\chi\left(\frac{\operatorname{dist}(x, \partial \Omega)}{\varepsilon}\right)\left(\psi(x)-\psi^{i}\right)\right) & \text { при } x \in \Gamma_{2 \varepsilon}^{i}, \\ w(x) & \text { при } x \in \bar{\Omega} \backslash \bigcup_{i} \Gamma_{2 \varepsilon}^{i},\end{cases}
$$

где $\chi$ такое же, как в односвязном случае, мы получаем корректно определенную $w_{\varepsilon}(x)$. Выбрав $w_{\varepsilon}$ таким образом, мы можем использовать те же рассуждения, что и выше.

ЗАмечАниЕ 11. Теорема 4 показывает, что в кольце с гладкими начальными данными вращательного типа не может быть диких решений со свойством Гёльдера. Действительно, любое допустимое решение со свойством Гёльдера диссипативно в силу нашей теоремы, так что оно должно совпадать со стационарным решением в силу единственности сильных решений в классе слабых. Это наблюдение представляет особый интерес в свете недавних результатов (см., например, [6], [9], [19]), в которых строятся примеры диких решений со свойством Гёльдера.

Одна из первых работ профессора М. И. Вишика "Об общих краевых задачах для эллиптических дифференииальных уравнений" [33] оказала, особенно во Франиии, существенное влияние на подготовку поколения математиков, к которому принадлежит первый автор настоящей публикации. Когда вслед за тем М.И. Вишик обратился к уравнениям Навъе-Стокса и турбулентности, он сыграл значительную роль в прогрессе, произошедшем за последние 60 лет в понимании математики турбулентных процессов в гидродинамике. Мы надеемся, что наше эссе внесет свой вклад в сохранение памяти о М.И. Вишике и в признание его влияния на математическое сообщество.

Авторы благодарны профессору Эдриссу Тити за интересные и плодотворные обсуждения.

\section{Список литературы}

[1] C. J. Amick, "Existence of solutions to the nonhomogeneous steady Navier-Stokes equations", Indiana Univ. Math. J., 33:6 (1984), 817-830.

[2] К. Бардос, Э. С. Тити, "Уравнения Эйлера идеальной несжимаемой жидкости", УМH, 62:3(375) (2007), 5-46; англ. пер.: C. Bardos, E. S. Titi, "Euler equations for incompressible ideal fluids", Russian Math. Surveys, 62:3 (2007), 409-451.

[3] C. Bardos, E. S. Titi, Mathematics and turbulence: where do we stand?, 2013, 40 pp., arXiv: 1301.0273 .

[4] C. Bardos, E. S. Titi, E. Wiedemann, "The vanishing viscosity as a selection principle for the Euler equations: the case of 3D shear flow", C. R. Math. Acad. Sci. Paris, 350:15-16 (2012), 757-760.

[5] Y. Brenier, C. De Lellis, L. Székelyhidi, Jr., "Weak-strong uniqueness for measurevalued solutions", Comm. Math. Phys., 305:2 (2011), 351-361.

[6] T. Buckmaster, C. De Lellis, L. Székelyhidi, Transporting microstructure and dissipative Euler flows, 2013, 35 pp., arXiv: 1302.2815.

[7] E. Chiodaroli, C. De Lellis, O. Kreml, Global ill-posedness of the isentropic system of gas dynamics, 2013, 30 pp., arXiv: 1304.0123. 
[8] C. M. Dafermos, Hyperbolic conservation laws in continuum physics, 3rd ed., Grundlehren Math. Wiss., 325, Springer-Verlag, Berlin, 2010, xxxvi+708 pp.

[9] S. Daneri, Cauchy problem for dissipative Hölder solutions to the incompressible Euler equations, 2013, 33 pp., arXiv: 1302.0988.

[10] C. De Lellis, L. Székelyhidi, Jr., "The Euler equations as a differential inclusion", Ann. of Math. (2), 170:3 (2009), 1417-1436.

[11] C. De Lellis, L. Székelyhidi, Jr., "On admissibility criteria for weak solutions of the Euler equations", Arch. Ration. Mech. Anal., 195:1 (2010), 225-260.

[12] C. De Lellis, L. Székelyhidi, Jr., "The $h$-principle and the equations of fluid dynamics", Bull. Amer. Math. Soc. (N. S.), 49:3 (2012), 347-375.

[13] J. Duchon, R. Robert, "Inertial energy dissipation for weak solutions of incompressible Euler and Navier-Stokes equations", Nonlinearity, 13:1 (2000), 249-255.

[14] L. C. Evans, Partial differential equations, 2nd ed., Grad. Stud. Math., 19, Amer. Math. Soc., Providence, RI, 2010, xxii+749 pp.

[15] G. L. Eyink, K. R. Sreenivasan, "Onsager and the theory of hydrodynamic turbulence", Rev. Modern Phys., 78:1 (2006), 87-135.

[16] G.P. Galdi, An introduction to the mathematical theory of the Navier-Stokes equations, v. I: Linearized steady problems, Springer Tracts Natur. Philos., 38, SpringerVerlag, New York, 1994, xii+450 pp.

[17] G. P. Galdi, "An introduction to the Navier-Stokes initial-boundary value problem", Fundamental directions in mathematical fluid mechanics, Adv. Math. Fluid Mech., Birkhäuser, Basel, 2000, 1-70.

[18] Д. Гилбарг, Н.С. Трудингер, Эллиптические дифференциалъные уравнения с частными производными второго порлдка, Наука, М., 1989, 464 с.; пер. с англ.: D. Gilbarg, N.S. Trudinger, Elliptic partial differential equations of second order, reprint of the 1998 edition, Classics Math., Springer-Verlag, Berlin, 2001, xiv+517 pp.

[19] P. Isett, Hölder continuous Euler flows in three dimensions with compact support in time, 2012 (v4 - 2014), 176 pp., arXiv: 1211.4065.

[20] T. Kato, "On classical solutions of the two-dimensional non-stationary Euler equation", Arch. Ration. Mech. Anal., 25:3 (1967), 188-200.

[21] T. Kato, "Remarks on zero viscosity limit for nonstationary Navier-Stokes flows with boundary", Seminar on nonlinear partial differential equations (Berkeley, CA, 1983), Math. Sci. Res. Inst. Publ., 2, Springer, New York, 1984, 85-98.

[22] P.-L. Lions, Mathematical topics in fluid mechanics, v. 1: Incompressible models, Oxford Lecture Ser. Math. Appl., 3, The Clarendon Press, Oxford Univ. Press, New York, 1996, xiv+237 pp.

[23] M. C. Lopes Filho, H. J. Nussenzveig Lopes, Yuxi Zheng, "Convergence of the vanishing viscosity approximation for superpositions of confined eddies", Comm. Math. Phys., 201:2 (1999), 291-304.

[24] A. J. Majda, A. L. Bertozzi, Vorticity and incompressible flow, Cambridge Texts Appl. Math., 27, Cambridge Univ. Press, Cambridge, 2002, xii+545 pp.

[25] L. Saint-Raymond, "Convergence of solutions to the Boltzmann equation in the incompressible Euler limit", Arch. Ration. Mech. Anal., 166:1 (2003), 47-80.

[26] V. Scheffer, "An inviscid flow with compact support in space-time", J. Geom. Anal., 3:4 (1993), 343-401.

[27] A. I. Shnirelman, "Lattice theory and flows of ideal incompressible fluid", Russian J. Math. Phys., 1:1 (1993), 105-114.

[28] A. Shnirelman, "On the nonuniqueness of weak solution of the Euler equation", Comm. Pure Appl. Math., 50:12 (1997), 1261-1286.

[29] A. Shnirelman, "Weak solutions with decreasing energy of incompressible Euler equations", Comm. Math. Phys., 210:3 (2000), 541-603. 
[30] L. Székelyhidi, Jr., "Weak solutions to the incompressible Euler equations with vortex sheet initial data", C. R. Math. Acad. Sci. Paris, 349:19-20 (2011), 1063-1066.

[31] L. Székelyhidi, Jr., From isometric embeddings to turbulence, Lecture note № 41, MaxPlanck-Institut für Mathematik in den Naturwissenschaften, Leipzig, 2012, 54 pp., http://www.mis.mpg.de/preprints/ln/lecturenote-4112.pdf.

[32] L. Székelyhidi, Jr., E. Wiedemann, "Young measures generated by ideal incompressible fluid flows", Arch. Ration. Mech. Anal., 206:1 (2012), 333-366.

[33] М.И. Вишик, "Об общих краевых задачах для эллиптических дифференциальных уравнений", Тр. ММО, 1, ГИТТЛ, М.-Л., 1952, 187-246; англ. пер.: M. I. Vishik, "On general boundary problems for elliptic differential equations", Eight papers on differential equations, Amer. Math. Soc. Transl. Ser. 2, 24, Amer. Math. Soc., Providence, RI, 1963, 107-172.

[34] E. Wiedemann, "Existence of weak solutions for the incompressible Euler equations", Ann. Inst. H. Poincaré Anal. Non Linéaire, 28:5 (2011), 727-730.

Клод Бардос

(Claude Bardos)

Université Paris VII - Denis Diderot, Paris, France

E-mail: claude.bardos@gmail.com

\section{Ласло Секелихиди мл.}

(Laszlo Szekelyhidi, Jr.)

Universität Leipzig, Mathematisches Institut,

Leipzig, Germany

E-mail: szekelyhidi@math.uni-leipzig.de

\section{Эмиль Видеманн}

\section{(Emil Wiedemann)}

University of British Columbia, Vancouver, Canada;

Pacific Institute for the Mathematical Sciences,

Vancouver, Canada

E-mail: emil@math.ubc.ca
Поступила в редакцию

27.10 .2013 